\title{
Inequalities for generalized divisor functions
}

\author{
József Sándor \\ Department of Mathematics, Babeş-Bolyai University \\ Str. Koğalniceanu 1, 400084 Cluj-Napoca, Romania \\ e-mail: jsandor@math.ubbcluj.ro
}

Received:19 December 2020

Revised: 20 April 2021

Accepted: 1 May 2021

Abstract: We offer inequalities to $\sigma_{a}(n)$ as a function of the real variable $a$. Monotonicity and convexity properties to this and related functions are proved, too. Extensions and improvements of known results are provided.

Keywords: Arithmetic functions, Inequalities for arithmetic functions, Monotonicity and convexity of real functions, Inequalities for sums, Series and integrals.

2020 Mathematics Subject Classification: 11A25, 26D07, 26D15, 26A51.

\section{Introduction}

Let $n \geq 1$ be a positive integer, and $a$ be a real variable. The sum of $a$-th powers of divisors of $n$ is defined by

$$
\sigma_{a}(n)=\sum_{d \mid n} d^{a}
$$

where $d$ runs through all distinct positive divisors of $n$. Particularly, $\sigma_{1}(n)=\sigma(n)$ is the sum of divisors of $n$, and $\sigma_{0}(n)=d(n)=$ number of distinct divisors of $n$. Remark that

$$
\sigma_{-1}(n)=\sum_{d \mid n} \frac{1}{d}=\frac{1}{n} \cdot \sum_{d \mid n} \frac{n}{d}=\frac{1}{n} \sum_{d \mid n} d=\frac{\sigma(n)}{n} .
$$

Similarly,

$$
\sigma_{-a}(n)=\frac{\sigma_{a}(n)}{n^{a}}
$$

for any real number $a$. It is well-known that $\left(\sigma_{a}(n)\right)_{n}$ is a multiplicative function of the natural variable $n$, i.e.,

$$
\sigma_{a}(n \cdot m)=\sigma_{a}(n) \cdot \sigma_{a}(m)
$$

for any $n, m \geq 1 ;(n, m)=1$. 
In other words, if $n=\prod_{i=1}^{r} p_{i}^{a_{i}}(r \geq 1)$ is the prime factorization of $n$, then

$$
\sigma_{a}(n)=\prod_{i=1}^{r} \sigma_{a}\left(p_{i}^{a_{i}}\right)=\prod_{i=1}^{r} \frac{p_{i}^{a\left(a_{i}+1\right)}-1}{p_{i}^{a}-1} .
$$

The unitary sum of divisor function $\sigma_{a}^{*}(n)$ is defined as

$$
\sigma_{a}^{*}=\sum_{d \mid n,(d, n / d)=1} d^{a}
$$

i.e., the sum of $a$-th powers of the unitary divisors of $n$, where $d \mid n$ is a unitary divisor of $n$, if $(d, n / d)=1$. It is well-known also (see e.g. $[2,3,6])$ that $\left(\sigma_{a}^{*}(n)\right)_{n}$ is a multiplicative function of $n$, i.e., fot the above prime factorization of $n$ one has

$$
\sigma_{a}^{*}(n)=\prod_{i=1}^{r} \sigma_{a}^{*}\left(p_{i}^{a_{i}}\right)=\prod_{i=1}^{r}\left(p_{i}^{a \cdot a_{i}}+1\right) .
$$

There are many known inequalities for $\sigma_{a}(n)$ and $\sigma_{a}^{*}(n)$, when $a=1$ or $a=k=$ positive integer. For example, a result of Sándor-Tóth [2] states that

$$
\frac{\sigma_{k}(n)}{d(n)}>n^{k / 2}
$$

for $n>1, k \geq 1$ integers. A result by the author [5] states that

$$
\frac{\sigma_{k}(n)}{\sigma_{k}^{*}(n)}<\frac{d(n)}{d^{*}(n)}
$$

In what follows, we will obtain extensions and refinements of (7), (8); and many related inequalities will be offered. For other inequalities, see [7].

\section{Monotonocity and convexity properties}

Theorem 1. The applications $f, g: \mathbb{R} \rightarrow \mathbb{R}_{+}$, defined by $f(a)=\sigma_{a}(n)$ and $g(a)=\sigma_{a}^{*}(n)$ are log-convex functions for any fixed integer $n \geq 1$.

Proof. The log-convexity of the function $f(a)$ means that the function $F(a)=\log f(a)$ is convex. It is well-known, that for continuous functions, a function is convex iff it is Jensen-convex. As $f(a)$ is sum of continuous functions, clearly it is continuous, too. Thus, we have to prove that $\log f(a)$ is Jensen-convex, i.e.,

$$
\log f\left(\frac{a+b}{2}\right) \leq \frac{\log f(a)+\log f(b)}{2}
$$

or equivalently

$$
f^{2}\left(\frac{a+b}{2}\right) \leq f(a) \cdot f(b)
$$

where $a, b$ are real numbers. 
In order to prove (10), we apply the Cauchy-Bunyakovsky inequality (see [1])

$$
\left(\sum_{i=1}^{r} x_{i} y_{i}\right)^{2} \leq\left(\sum_{i=1}^{r} x_{i}^{2}\right) \cdot\left(\sum_{i=1}^{r} y_{i}^{2}\right)
$$

for $x_{i}=d_{i}^{a / 2}, y_{i}=d_{i}^{b / 2}$, where $1=d_{1}<d_{2}<\cdots<d_{r}=n$ are the distinct divisors of $n$. As

$$
\sum_{i=1}^{n} d_{i}^{a}=f(a), \quad \sum_{i=1}^{r} d_{i}^{b}=f(b), \quad \sum_{i=1}^{r} d_{i}^{(a+b) / 2}=f((a+b) / 2),
$$

by (11) we get relation (10).

Applying the same inequality to $x_{i}=\left(d_{i}^{*}\right)^{a / 2}, y_{i}\left(d_{i}^{*}\right)^{b / 2}$, where $1 \leq d_{1}^{*}<d_{2}^{*}<\cdots<d_{r}^{*}=n$ are the distinct unitary divisors of $n$, we get

$$
g^{2}\left(\frac{a+b}{2}\right) \leq g(a) \cdot g(b)
$$

i.e., the function $g(a)$ will be log-convex, too.

Remark 1. As there is equality in (11) only if $\left(x_{i}\right)$ and $\left(y_{i}\right)$ are proportional, i.e. $x_{i} / y_{i}=\lambda$ $(i=1,2, \ldots, r) \lambda=$ constant, clearly there is an equality in (10) only for $a=b$.

\section{Corollary 1.}

$$
\left[\sigma_{(a+b) / 2}(n)\right]^{2} \leq \sigma_{a}(n) \sigma_{b}(n) \leq\left[\frac{\sigma_{a}(n)+\sigma_{b}(n)}{2}\right]^{2} .
$$

The sequence of general term

$$
t_{k}=\frac{\sigma_{k}(n)}{\sigma_{k-1}(n)} \quad(k \geq 1)
$$

is strictly increasing for any fixed $n>1$.

- Indeed, the second inequality of (12) follows by $x y \leq\left(\frac{x+y}{2}\right)^{2}$, where $x=\sigma_{a}(n), y=\sigma_{b}(n)$.

For $a=k-1, b=k+1$ we set from (12) that $\left(\sigma_{k}(n)\right)^{2}<\sigma_{k-1}(n) \cdot \sigma_{k+1}(n) ;$ i.e. $t_{k}<t_{k+1}$.

\section{Corollary 2.}

$$
n^{a / 2} \leq \frac{\sigma_{a}(n)}{d(n)} \leq \frac{\sigma_{2 a}(n)}{\sqrt{d(n)}}
$$

for any $a \in \mathbb{R}$.

Indeed, let $b=-a$ in (12). By relation (2) we get the left-hand side of (13).

Let now $a \rightarrow a+b, b \rightarrow a-b$ in the left-hand side of (12). We get the inequality

$$
\left(\sigma_{a}(n)\right)^{2} \leq \sigma_{a+b}(n) \cdot \sigma_{a-b}(n) .
$$

By letting $b=a$ in (14), we get the right-hand side of (13).

Remark 2. All the above inequalities (12)-(14) hold true also for $\sigma_{a}^{*}(n)$ in plane of $\sigma_{a}(n)$, etc.

Theorem 2. The functions $F, G:(0, \infty) \rightarrow \mathbb{R}$ defined by

$$
F(a)=\sigma_{a}(n){ }_{n}^{a / 2} ; \quad G(a)=\sigma_{a}^{*}(n) / \frac{a / 2}{n}
$$

are strictly increasing functions. The functions $F_{1}, G_{1}:(-\infty, 0) \rightarrow \mathbb{R}$ with the same definitions as above, are strictly decreasing. 
Proof. Let $p$ be a prime number. As $F\left(p^{\alpha}\right)=\sigma_{a}\left(p^{\alpha}\right) / p^{a \alpha / 2}=s(a)$, we will prove first that $s(a)$ is strictly increasing. Then, as

$$
F(a)=\prod_{i=1}^{r} \frac{\sigma_{a}\left(p_{i}^{\alpha_{i}}\right)}{p_{i}^{a \alpha_{i} / 2}}
$$

$F(a)$ will be strictly increasing as the product of strictly increasing positive functions.

By (4) one has

$$
\log s(a)=\log \left(p^{a(\alpha+1)}-1\right)-\log \left(p^{a}-1\right)-\frac{a \alpha}{2} \log p=S(a) .
$$

One has for the derivative of $S(a)$ that

$$
S^{\prime}(a)=\frac{(\alpha+1) p^{a(\alpha+1)} \log p}{p^{a(\alpha+1)}-1}-\frac{p^{a} \log p}{p^{a}-1}-\frac{\alpha}{2} \log p .
$$

By letting $p^{a}=x$, after some elementary computations, we get

$$
S^{\prime}(a) \cdot \frac{2(x-1) \cdot\left(x^{\alpha+1}-1\right)}{\log p}=x^{\alpha+2} \cdot(\alpha)-x^{\alpha+1} \cdot(\alpha+2)+x \cdot(\alpha+2)-\alpha=M(x) .
$$

Now, remark that $M(1)=0$,

$$
M^{\prime}(x)=(\alpha+2) \cdot\left[\alpha \cdot x^{\alpha+1}-(\alpha+1) \cdot x^{\alpha}+1\right]=(\alpha+2) \cdot N(x) .
$$

Here $N(1)=0$ and $N^{\prime}(x)=(\alpha+1) \cdot x^{\alpha-1} \cdot(x-1)>0$, as $x=p^{a}>1, \alpha \geq 1$. Therefore, $N(x)$ is strictly increasing, implying $N(x)>N(1)=0$, so $M^{\prime}(x)>0$. Thus, finally, we get $M(x)>M(1)=0$, so $S^{\prime}(a)>0$, and thus $S(a)$ is strictly increasing. This means that $s(a)$ is strictly increasing, and the first part of Theorem 2 is proved.

For the second part, remark that $\sigma_{a}^{*}\left(p^{\alpha}\right)=p^{a \alpha}+1$, and it will be sufficient to consider the monotonicity of

As

$$
\log \left(p^{a^{\alpha}}+1\right)-\frac{a \alpha}{2} \log p=h(a)
$$

$$
\frac{h^{\prime}(a)}{\alpha \log p}=\frac{p^{a \alpha}}{p^{a \alpha}+1}-\frac{1}{2}=\frac{x^{\alpha}-1}{2\left(x^{\alpha}+1\right)},
$$

where $x=p^{a}>1$ for $a>0$. Clearly $x^{\alpha}-1>0$, so $h^{\prime}(a)>0$, and the proof of second part of the theorem follows. For $a<0$ we get $0<x<1$, and all can be replaced for $F_{1}$ and $G_{1}$, will be strictly decreasing.

\section{Corollary 3.}

$$
\frac{\sigma_{a}(n)}{d(n)}>n^{a / 2} \text { for } n>1, a \neq 0
$$

As

$$
\lim _{a \rightarrow 0} \frac{\sigma_{a}\left(p^{\alpha}\right)}{p^{a \alpha / 2}}=\lim _{a \rightarrow 0} \frac{p^{a(\alpha+1)}-1}{p^{a}-1}=\alpha+1
$$

(by L'Hospital's rule) and as

$$
F(a)>\lim _{a \rightarrow 0} f(a)=\left(\alpha_{1}+1\right) \cdots\left(\alpha_{r}+1\right)=d(n),
$$

relation (16) follows from the first part of Theorem 2. From $F_{1}(a)>\lim _{a \rightarrow 0} F_{1}(a)$ for $a<0$, we get the same inequalities. 


\section{Corollary 4.}

$$
\frac{\sigma_{a}^{*}(n)}{d(n)}>n^{a / 2} \text { for } n>1, a \neq 0 .
$$

This follows in a similar manner, from the second part of Theorem 2, first for $G(a)$, then for $G_{1}(a)$.

Theorem 3. The function $H:(0, \infty) \rightarrow \mathbb{R}$, defined by

$$
H(a)=\sigma_{a}(n) / \sigma_{a}^{*}(n)(n>1 \text { fixed })
$$

is strictly decreasing. The function $H_{1}:(-\infty, 0) \rightarrow \mathbb{R}$ with the same definition is strictly increasing.

Proof. For $n=p_{1}^{\alpha_{1}} \cdots p_{r}^{\alpha_{r}}$ one has

$$
H(n)=\sigma_{a}\left(p_{1}^{\alpha_{1}}\right) \cdots \sigma_{a}\left(p_{r}^{\alpha_{r}}\right) / \sigma_{a}^{*}\left(\sigma_{a}^{p_{1}}\right) \cdots \sigma_{a}^{*}\left(p_{r}^{\alpha_{1}}\right)=f_{1}(a) \cdots f_{r}(a)
$$

where $f_{i}(a)=\sigma_{a}\left(p_{i}^{\alpha_{i}}\right) / \sigma_{a}^{*}\left(p_{i}^{\alpha_{i}}\right)$, so it will be sufficient to prove that

$$
k(a)=\sigma_{a}\left(p^{\alpha}\right) / \sigma_{a}^{*}\left(p^{\alpha}\right)=\left[p^{a(\alpha+1)}-1\right] /\left(p^{a}-1\right)\left(p^{a \alpha}+1\right)
$$

will be strictly decreasing for fixed prime $p>1$. One has

$$
\log k(a)=\log \left(p^{a(\alpha+1}-1\right)-\log \left(p^{a}-1\right)-\log \left(p^{a \alpha+1}+1\right)=K(a) .
$$

One has

$$
\frac{K^{\prime}(a)}{p^{a} \log p}=\frac{(a+1) \cdot p^{a \alpha}}{p^{a(\alpha+1)}-1}-\frac{1}{p^{a}-1}-\frac{\alpha p^{a(\alpha+1)}}{p^{a \alpha}+1},
$$

and after some elementary computations we can write

$$
\begin{aligned}
& K^{\prime}(a) \cdot \frac{\left[p^{a(\alpha+1)}-1\right]\left(p^{a}-1\right)\left(p^{a \alpha}+1\right)}{p^{a} \log p} \\
& =(\alpha+1) x^{\alpha} \cdot(x-1) \cdot\left(x^{\alpha}+1\right)-\left(x^{\alpha}+1\right) \cdot\left(x^{\alpha+1}-1\right)-\alpha \cdot x^{\alpha-1} \cdot(x-1) \cdot\left(x^{\alpha+1}-1\right) \\
& =R(x),
\end{aligned}
$$

where $x=p^{a}>1$. Now, $R(x)$ can be written as $R(x)=\alpha \cdot x^{\alpha+1}-x^{2 \alpha}-\alpha \cdot x^{\alpha-1}+1$. We will prove that $-R(x)=x^{2 \alpha}-\alpha \cdot x^{\alpha+1}+\alpha \cdot x^{\alpha-1}-1 \geq 0$. One has

$$
-R^{\prime}(x)=\alpha \cdot x^{\alpha-2} \cdot\left[2 x^{\alpha+1}-(\alpha+1) x^{2}+\alpha-1\right] .
$$

Let $U(x)=2 x^{\alpha+1}-(\alpha+1) x^{2}+\alpha-1$. Here $U(1)=0$ and $U^{\prime}(x)=2(\alpha+1) \cdot x \cdot\left(x^{\alpha-1}-1\right) \geq 0$ as $x>1$ and $\alpha-1 \geq 0$. Thus $U(x)>U(1)=0$, so we get $R^{\prime}(x)<0$ implying $R(x)<R(1)=0$. Thus we have proved that $K^{\prime}(a)<0$. As $\frac{k^{\prime}(a)}{k(a)}=K^{\prime}(a)$, this implies finally that $k^{\prime}(a)<0$, i.e., $k(a)$ is strictly decreasing. For $a<0$ one has $0<x<1$, and we get that $H_{1}(x)$ is strictly increasing. 
Corollary 5. For any $a \neq 0$ one has

$$
\frac{\sigma_{a}(n)}{\sigma_{a}^{*}(n)}<\frac{d(n)}{d^{*}(n)}
$$

Indeed, as

$$
H(a)<\lim _{a \rightarrow 0+} H(a)=\frac{\alpha+1}{2}
$$

by Theorem 3 we can write

$$
\frac{\sigma_{a}(n)}{\sigma_{a}^{*}(n)}<\frac{\alpha_{1}+1}{2} \cdots \frac{\alpha_{r}+1}{2}=\frac{d(n)}{d^{*}(n)} .
$$

From $H_{1}(a)<\lim _{a \rightarrow 0-} H_{1}(a)$ we get the same inequality.

Remark 3. Inequality (18) extends (8) from positive integers $k$ to all real numbers $a \neq 0$. Finally, in this context, we will prove:

Theorem 4. The function $T(a):(0, \infty) \rightarrow \infty$, defined by

$$
T(a)=\frac{\sigma_{a}(n)}{\sigma_{a}^{*}(\gamma(n))},
$$

(where $\gamma(n)$ is the "core" of $n$ ) is strictly increasing. The function $T_{1}(a):(-\infty, 0) \rightarrow \mathbb{R}$ with the same definition is strictly decreasing.

Proof. If $n=p_{1}^{\alpha_{1}} \cdots p_{1}^{\alpha_{r}}$, then $\gamma(n)=p_{1} \cdots p_{r}$; so $\sigma_{a}^{*}(\gamma(n))=\left(p_{1}^{a}+1\right) \cdots\left(p_{r}^{a}+1\right)$. Thus, it will be sufficient to prove that the function $z(a)=\sigma_{a}\left(p^{\alpha}\right) / p^{a}+1$ will be strictly increasing. As

$$
\log z(a)=\log \left(p^{a(\alpha+1)}-1\right)-\log \left(p^{a}-1\right)-\log \left(p^{a}+1\right)=Z(a),
$$

one has

$$
Z^{\prime}(a)=\frac{(\alpha+1) \cdot p^{a(\alpha+1)} \log p}{p^{a(\alpha+1)}-1}-\frac{p^{a} \log p}{p^{a}-1}-\frac{p^{a} \log p}{p^{a}+1}
$$

and after some elementary computations (which we omit here) we can find that

$$
\frac{\left(p^{a(\alpha+1}-1\right)\left(p^{a}-1\right)\left(p^{a}+1\right) Z^{\prime}(a)}{\log p}=x^{2} \cdot\left[(\alpha-1) \cdot x^{\alpha+1}-(\alpha+1) \cdot x^{\alpha-1}+2\right],
$$

where $x=p^{\alpha}>1$. Let $q(x)=(\alpha-1) \cdot x^{\alpha+1}-(\alpha+1) x^{\alpha-1}+2$. We have $q(1)=0$ and $q^{\prime}(x)=\left(\alpha^{2}-1\right) \cdot x^{\alpha-2} \cdot\left(x^{2}-1\right) \geq 0$ as $x>1$ and $\alpha \geq 1$. Thus $q(x)>q(1)=0$ and this yields $Z^{\prime}(a)>0$, so $z(a)$ will be strictly increasing. For $a<0$ we have $0<x<1$, and we get that $T_{1}(a)$ is strictly decreasing.

Corollary 6. For any $a \neq 0$ and $n>1$ one has

$$
\frac{d(n)}{d^{*}(n)}<\frac{\sigma_{a}(n)}{\sigma_{a}^{*}(\gamma(n))}
$$

This follows by

$$
\lim _{a \rightarrow 0} T(a)=\frac{d(n)}{d^{*}(a)}
$$

and Theorem 4.

Remark 4. Inequality (20) offers a counterpart to (18). 


\section{Applications of other inequalities for sums}

The classical Chebyshev inequality (see [1]) states that if $\left(x_{i}\right)$ and $\left(y_{i}\right)(i=1,2, \ldots, r)$ are two sequences with the same (reversed) type of monotonicity, then

$$
\frac{x_{1} y_{1}+\cdots+x_{r} y_{r}}{r} \underset{(\leq)}{\geq} \frac{x_{1}+\cdots+x_{r}}{r} \cdots \frac{y_{1}+\cdots+y_{r}}{r} .
$$

Letting $x_{i}=d_{i}^{a}, y_{i}=d_{i}^{b}$, where $1=d_{1}<d_{2}<\cdots<d_{r}=n$ are all divisors of $n$, we get the following:

$$
\begin{aligned}
& d(n) \cdot \sigma_{a+b}(n) \geq \sigma_{a}(n) \sigma_{b}(n) \quad \text { for } a \cdot b \geq 0, \\
& d(n) \cdot \sigma_{a+b}(n) \leq \sigma_{a}(n) \sigma_{b}(n) \quad \text { for } a \cdot b \leq 0 .
\end{aligned}
$$

Indeed, for $a \cdot b \geq 0$, the sequences $\left(d_{i}^{a}\right)$ and $\left(d_{i}^{b}\right)$ will have the same type of monotonicity, and for $a \cdot b \leq 0$, the reversed one.

From the left-hand side of (12), combined with (23), we get:

$$
\left[\sigma_{(a+b) / 2}(n)\right]^{2} \leq \sigma_{a}(n) \sigma_{b}(n) \leq d(n) \cdot \sigma_{a+b} \quad \text { for } a \cdot b \geq 0 .
$$

Particularly, by letting $\frac{a+b}{2}=c$, the weakest part of (24) offers $\left(\sigma_{c}(n)\right)^{2} \leq d(n) \sigma_{2 c}(n)$, which is the right-hand side of (13). Thus, (24) offers an improvement of right-hand side of (13) for $a \cdot b \geq 0$.

The Milne's inequality (see $[1,4])$ states that if $\left(x_{i}\right)$ and $\left(y_{i}\right)$ are positive $r$-tuples, then

$$
\sum_{i=1}^{r}\left(x_{i}+y_{i}\right) \cdot \sum_{i=1}^{r} \frac{x_{i} y_{i}}{x_{i}+y_{i}} \leq \sum_{i=1}^{r} x_{i} \cdot \sum_{i=1}^{r} y_{i}
$$

with equality if and only if $\left(x_{i}\right)$ and $\left(y_{i}\right)$ are proportional.

Apply now the Cauchy-Bunyakovsky inequality (11) for $x_{i}=\sqrt{a_{i}^{2}+b_{i}^{2}}, y_{i}=\frac{a_{i} b_{i}}{\sqrt{a_{i}^{2}+b_{i}^{2}}}$. We get

$$
\left(\sum_{i=1}^{r} a_{i} b_{i}\right)^{2} \leq \sum_{i=1}^{r}\left(a_{i}^{2}+b_{i}^{2}\right) \cdot \sum_{i=1}^{r} \frac{a_{i}^{2} b_{i}^{2}}{a_{i}^{2}+b_{i}^{2}} .
$$

Now, Milne's inequality (25) applied for $x_{i}=a_{i}^{2}, y_{i}=b_{i}^{2}$ and combined with (26) gives

$$
\left(\sum_{i=1}^{r} a_{i} b_{i}\right)^{2} \leq \sum_{i=1}^{r}\left(a_{i}^{2}+b_{i}^{2}\right) \cdot \sum_{i=1}^{r} \frac{a_{i}^{2} b_{i}^{2}}{a_{i}^{2}+b_{i}^{2}} \leq\left(\sum_{i=1}^{r} a_{i}^{2}\right)\left(\sum_{i=1}^{r} b_{i}^{2}\right)
$$

where $a_{i}, b_{i}$ are real numbers and $a_{i}^{2}+b_{i}^{2} \neq 0$. This is in fact a refinement of Cauchy-Bunyakovsky inequality. Let now $a_{i}=d_{i}^{a / 2}, b_{i}=d_{i}^{b / 2}$, where $1=d_{1}<d_{2}<\cdots<d_{r}=n$ are the distinct divisors of $n$. We get the following refinement of the left-hand side of (12):

$$
\left(\sigma_{(a+b) / 2}(n)\right)^{2} \leq A(a, b, n) \leq \sigma_{a}(n) \cdot \sigma_{b}(n),
$$

where

$$
A(a, b, n)=(\sigma(a)+\sigma(b)) \cdot \sum_{d \mid n} \frac{d^{a+b}}{d^{a}+d^{b}} .
$$

The Pólya-Szegó inequality (see [1]) states that if $0<a \leq x_{i} \leq A$ and $0<b \leq y_{i} \leq B$ $(i=1,2, \ldots, r)$. 
Then

$$
\left(\sum_{i=1}^{r} x_{i}^{2}\right)^{1 / 2}\left(\sum_{i=1}^{r} y_{i}^{2}\right)^{1 / 2} \leq \frac{1}{2}\left(\sqrt{\frac{A B}{a b}}+\sqrt{\frac{a b}{A B}}\right) \sum_{i=1}^{r} x_{i} y_{i} .
$$

Let $x_{i}=d_{i}^{a / 2}, y_{i}=d_{i}^{b / 2}\left(d_{i}\right.$ the divisors of $\left.n\right)$. After some elementary computations, we get:

$$
\begin{aligned}
& \sigma_{a}(n) \sigma_{b}(n) \leq \frac{1}{4} \cdot \frac{\left(n^{\frac{a+b}{2}}+1\right)^{2}}{n^{(a+b) / 2}} \cdot\left(\sigma_{(a+b) / 2}(n)\right)^{2} \quad \text { if } \quad a \cdot b>0, \\
& \sigma_{a}(n) \sigma_{b}(n) \leq \frac{1}{4} \cdot \frac{\left(n^{a / 2}+n^{b / 2}\right)^{2}}{n^{(a+b) / 2}} \cdot\left(\sigma_{(a+b) / 2}(n)\right)^{2} \quad \text { if } \quad a \cdot b<0 .
\end{aligned}
$$

These can complemenet the right-hand side of inequality (28).

Finally, the discrete version of Zagier's inequality (see [1]) states that

$$
\frac{\left(\sum_{i=1}^{r} x_{i}^{2}\right)\left(\sum_{i=1}^{r} y_{i}^{2}\right)}{\max \left\{\sum_{i=1}^{r} x_{i}, \sum_{i=1}^{r} y_{i}\right\}} \leq \sum_{i=1} x_{i} y_{i},
$$

where $0<x_{i}, y_{i} \leq 1$, where both of $\left(x_{i}\right)$ and $\left(y_{i}\right)$ are decreasing sequences.

For $x_{i}=d_{i}^{a / 2}, y_{i}=d_{i}^{b / 2}\left(d_{i}=\right.$ divisors of $\left.n\right)$, we get from (32):

$$
\frac{\sigma_{a}(n) \cdot \sigma_{b}(n)}{\max \left\{\sigma_{a / 2}(n), \sigma_{b / 2}(n)\right\}} \leq \sigma_{(a+b) / 2}(n) \quad \text { for } \quad a, b<0 .
$$

Letting $a=-A, b=-B$ and using (2) and (33), we get:

$$
\frac{\sigma_{A}(n) \cdot \sigma_{B}(n)}{\sigma_{B / 2}(n)} \leq n^{\frac{A}{2}} \cdot \sigma_{\frac{A+B}{A}}(n) \quad \text { for } \quad A \geq B>0 .
$$

\section{References}

[1] Bullen, P. (2015). Dictionary of Inequalities, second ed., CRC Press, Boca Raton, USA.

[2] Sándor, J., \& Tóth, L. (1990). On certain number theoretic inequalities. The Fibonacci Quarterly, 28(3), 255-258.

[3] Sándor, J., \& Tóth, L. (1997). On certain arithmetic functions associated with the unitary divisors of a number. Notes on Number Theory and Discrete Mathematics, 3(1), 1-8.

[4] Sándor, J. (2006). Generalizations of Lehman's inequality. Soochow Journal of Mathematics, 32(2), 301-309.

[5] Sándor, J. (2009). On the monotonicity of the sequence $\sigma_{k} / \sigma_{k}^{*}$. Notes on Number Theory and Discrete Mathematics, 15(3), 9-13.

[6] Sándor, J. (2014). On certain inequalities for $\sigma, \gamma, \psi$ and related functions. Notes on Number Theory and Discrete Mathematics, 20(2), 52-60.

[7] Sándor, J. \& Kovács, L. (2015). On certain upper bounds for the sum of divisors function $\sigma(n)$. Acta Universitatis Sapientiae, Mathematica, 7(2), 265-277. 